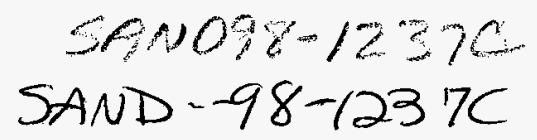

\title{
Electron cyclotron discharge cleaning (ECDC) experiments on Alcator C-Mod CONF-980560-
}

\author{
R.T. Nachtrieb, B.L. LaBombard, J.L Terry, J.C. Reardon, \\ W.L. Rowan ${ }^{1}$, W.R. Wampler ${ }^{2}$ \\ Plasma Science and Fusion Center, Massachusetts Institute of Technology, \\ Cambridge MA 02139, USA \\ ${ }^{1}$ Fusion Research Center, The University of Texas at Austin, Austin, TX, USA \\ ${ }^{2}$ Sandia National Laboratory, Albuquerque NM, USA
}

RECEIVED

JUN 089998

0.97

\begin{abstract}
Experiments were performed on Alcator C-Mod with Electron Cyclotron resonance plasmas to help determine their applicabilty to a fusion reactor. Strong radial inhomogeneity of the plasma density was measured, decreasing by a factor of ten a few centimeters inside the resonance location, but remaining approximately constant $\left(n_{e} \approx 10^{16} \mathrm{~m}^{-3}\right)$ outside the resonance location. Electron and ion temperatures remained mostly constant outside the resonance location $\left(T_{e} \approx 10 \mathrm{eV}, T_{i} \approx 2 \mathrm{eV}\right)$. Toroidal asymmetries in ion saturation current density were observed, indicating local toroidal plasma flow. The ECR plasma was used to remove a diamond-like carbon coating from a stainless-steel sample. Removal rates peaked at $4.2 \pm 0.4 \mathrm{~nm} /$ hour with the sample a few centimeters ouside the resonance location. Removal rates decreased inside and further outside the resonance location. The plasma did not remove the carbon from the sample uniformly, possibly due to plasma flow. Yields were calculated $\left(Y \approx 10^{-3}\right)$ to be lower than other published results for chemical sputtering of deuterium ions on carbon, possibly due to toroidally asymmetric plasma conditions. Significant redeposition was not observed.

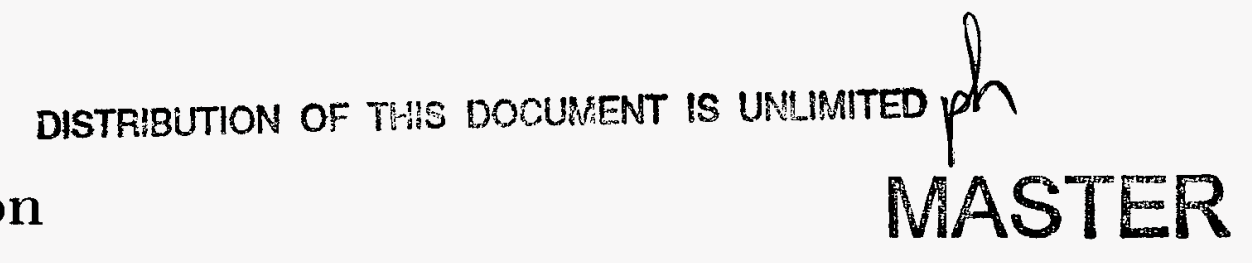

Experiments on magnetic fusion reactors have shown that conditioning of plasma-facing components affects performance. Electron cyclotron resonance heating of low density plasmas might be the only technique for conditioning the thick vessel walls of large fusion reactors with superconducting magnets. Electron cyclotron discharge cleaning
\end{abstract}


(ECDC) plasmas operate in steady state, require no inductive currents, and require only small port access for waveguides. Alcator C-Mod has demonstrated effective first-wall conditioning using ECDC [1].

To help determine if ECDC could be made to work for a fusion reactor, measurements were performed on ECDC plasmas on Alcator C-Mod to answer the following questions: (1) How do the plasma conditions vary with neutral pressure, (2) what is the spatial structure of the plasma, (3) how effectively do different radial zones of the plasma clean a test sample, and (4) is the mechanism for surface cleaning simply understood in termos of ion-induced chemical and physical sputtering processes?

ECDC plasmas were produced with magnetic fields with $.067 \leq B(\mathrm{~T}) \leq 0.11$ using the toroidal field coils only. The location of cyclotron resonance was swept between $.52-.83 \mathrm{~m}$ torus major radius by varying the magnetic field. Electromagnetic waves were launched from a horn at one toroidal location, with fixed frequency of $2.45 \mathrm{GHz}$ and power of $3 \mathrm{~kW}$. Here we report the results from experiments performed with deuterium fill gas with pressure between .04-.08 $\mathrm{Pa}$; we have also performed ECDC in helium.

\section{Experiments}

Figure 1 shows a poloidal projection of Alcator C-Mod indicating the diagnostics used for these experiments. Langmuir probes at the top (Omegatron) and bottom (FSP) of the vessel were used to measure plasma density and electron temperature at the same toroidal location as the microwave horn. Bulk plasma ion temperature was measured at the top of the torus with a retarding field energy analyzer. A CCD camera view from the top of the torus was used to give a qualitative picture of visible light emission. The probe group at the bottom of the machine consists (FSP) of four Langmuir probes, arranged on the faces of a pyramidal structure; see LaBombard [2] for details.

Asymmetries in the ion saturation current density to the different probes indicate local toroidal flow.

Approximately $20 \mathrm{~nm}$ of diamond-like carbon were deposited onto the end cylindrical stainless steel sample. The coating was estimated to be axially uniform to within $5 \mathrm{~nm}$, but was this was not verified before exposure to the plasma. A mask was 


\section{DISCLAIMER}

This report was prepared as an account of work sponsored by an agency of the United States Government. Neither the United States Government nor any agency thereof, nor any of their employees, makes any warranty, express or implied, or assumes any legal liability or responsibility for the accuracy, completeness, or usefulness of any information, apparatus, product, or process disclosed, or represents that its use would not infringe privately owned rights. Reference herein to any specific commercial product, process, or service by trade name, trademark, manufacturer, or otherwise does not necessarily constitute or imply its endorsement, recommendation, or favoring by the United States Government or any agency thereof. The views and opinions of authors expressed herein do not necessarily state or reflect those of the United States Government or any agency thereof. 
used to expose two areas of the coated sample to plasma facing toroidally clockwise and counterclockwise (as seen from the top). Figure Fig. 1 shows the coated sample mounted onto a scannable assembly, 180 degrees toroidally from the microwave horn. For the carbon-removal experiments, the radial location of the cyclotron resonance was fixed at $0.72 \mathrm{~m}$ and the coated sample moved into the plasma.

The thickness of the remaining coating was measured using Rutherford backscattering (RBS) of $1.7 \mathrm{MeV}$ protons. Comparison with uncoated region of the sample gave the thickness of carbon removed from by the plasma. An absolute error of 2 $\mathrm{nm}$ for the thickness measurements was determined by performing RBS measurements at identical locations using proton beams of $0.5 \mathrm{~mm}$ diameter and $1.0 \mathrm{~mm}$ diameter.

\section{Results}

\subsection{Plasma conditions, non-uniformities}

Fig. 2 shows plasma density, electron temperature, and ion temperature measured as a function of deuterium neutral pressure, for resonance location held fixed at $R=0.52 \mathrm{~m}$ and microwave power of $3 \mathrm{~kW}$. Results were obtained similar to Sakamoto et al [3], but ion temperatures are reported as well. A camera view from the top of the machine showed visible light emitted in a toroidally symmetric region, with a sharp boundary on the smaller major radius side of the resonance location. Decreasing the neutral deuterium pressure contracted the extent of a broader light-emitting region on the larger major radius side of the resonance location.

Figure Fig. 3 shows effective profiles of the plasma density and electron and ion temperatures, obtained by sweeping the location of the electron cyclotron resonance. The data presented in Fig. 3 do not represent actual plasma profiles since the probes were not moved relative to the plasma boundary, but the data demonstrate the lack of plasma source inside the resonance location and suggest outward plasma flux. Ion temperatures shown in Fig. 3 are measured in a local scrape-off plasma of the Omegatron; bulk plasma $T_{i}$ is likely to be higher.

Figure 4 shows effective radial profiles of saturation current density as measured by the Langmuir probes at the bottom of the machine. Note that the clockwise side of 
the probe receives higher ion saturation current density than the counterclockwise side by a factor of three, suggesting counterclockwise toroidal flow at the probe location.

Figure 4 also shows effective radial profiles of saturation current density as measured by the Langmuir probes at the top of the machine. The Langmuir probe at the top receives approximately the same plasma flux as the counterclockwise-facing probe at the bottom, confirming the vertical isotropy within a factor of two reported by Sharma et al [4].

\subsection{Surface erosion measurements}

Figure Fig. 5 shows the removal rates of diamond-like carbon by the ECDC plasma, revealing two significant trends: the plasma does not remove the coating evenly from each side of the sample, and the erosion rate depends on the location of the sample relative to the resonance.

Twice as much carbon is removed from the counterclockwise face of the sample as the clockwise face. Toroidal plasma flow at the bottom of the machine is inferred by the asymmetric ion saturation current densities to the Langmuir probe group there. If toroidal plasma flow exists at the location of the coated sample, albeit in the opposite direction, it could explain the asymmetry of the removal rates.

The plasma removed the most carbon coating when the sample was placed within 5 $\mathrm{cm}$ outside of the resonant surface; the removal rate decrease both when the sample was placed further outside the resonance than $5 \mathrm{~cm}$, and when the sample was placed inside the resonance. The effective yield of the plasma flux was calculated by $Y=n_{c} d /(\Gamma t)$, where $n_{c}=\rho_{c} / m_{c}$ represents the density of carbon deposited on the surface, $d / t$ represents the linear removal rate of carbon, and $\Gamma=n_{e} C_{s} / 2$ represents the ion flux to the surface. Using $n_{e}=1 \times 10^{16} \mathrm{~m}^{-3}, T_{e}=10 \mathrm{eV}, d / t=4 \mathrm{~nm} / \mathrm{hr}, \rho_{c}=4 \times 10^{3} \mathrm{~kg} / \mathrm{m}^{3}$ for diamond, and $m_{c}=12 \mathrm{~kg} / \mathrm{kmol}$ gives an effective yield of $Y=1 \times 10^{-3}$.

This calculated yield is lower than yields given in recent literature for chemical sputtering of deuterium on carbon, but higher than yields for physical sputtering. Mech [5] gives $Y=4 \times 10^{-2}$ for total chemical erosion yields of hydrogen of $25 \mathrm{eV}$ incident energy on carbon at $500 \mathrm{~K}$ surface temperature. The $25 \mathrm{eV}$ incident energy comes from assuming ions have only $3 k T_{e}$ sheath potential energy. Eckstein [6] predicts that $30 \mathrm{eV}$ monoenergetic deuterium ions bombarding carbon are below the threshold for physical 
sputtering, as is a distribution of deuterium ions with $T_{i}=2 \mathrm{eV}$. The plasma conditions reported here were not measured at the same toroidal location as the carbon-coated sample, yet toroidal symmetry was assumed in the calculation of the sputtering yield; discrepancy of our calculated yields with published results might be due to toroidal asymmetry of plasma conditions. Insufficient redeposition of carbon on the stainless steel mask was observed to account for the discrepancy. Significant redeposition is not expected, as neutral mean free paths are of order one meter in plasma conditions investigated here.

\section{Conclusions}

Experiments were performed on electron-cyclotron resonance plasmas on Alcator C-Mod. Camera views of the plasma showed toroidally symmetric visible light emission which decreased abruptly at a major radius near the cyclotron resonance, yet remained mostly uniform from the resonance location radially outward to the wall. Electron density measurements confirmed this radial structure: density decreased inside the resonant major radius by an order of magnitude over $10 \mathrm{~cm}$ while outside the resonance we recorded a uniform value of $n_{e} \approx 2 \times 10^{16} \mathrm{~m}^{-3}$. Typical electron temperatures were $T_{e} \approx 10 \mathrm{eV}$ outside the resonance, increasing by a factor of two slightly inside the resonance; uniform ion temperatures of $T_{i} \approx 2 \mathrm{ev}$ were measured. Toroidally asymmetric ion saturation current density measured at the bottom of the machine suggest toroidal plasma flow at that location.

The removal rate was measured of a diamond-like carbon coating on a stainless steel sample which was inserted into the ECDC plasma. By moving the exposed part of the sample relative to the resonance location, a coarse radial profile of the carbon removal rate was obtained. Results indicated maximum carbon removal rates $(4.2 \pm 0.4 \mathrm{~nm} /$ hour $)$ a few centimeters outside the resonance location. Removal rates decreased inside the resonance location and further outside the resonance location, indicating localized effective cleaning. Twice as much carbon was removed the side of the sample facing toroidally counterclockwise as the side facing clockwise. This asymmetry might be due to toroidal plasma flow. Plasma conditions and carbon 
removal rates give effective sputtering yield of $Y \leq 1 \times 10^{-3}$, less than yields predicted in the literature for chemical sputtering by similar plasmas.

\title{
5 Acknowledgements
}

Work supported by U.S. Department of Energy Contracts nos. DE-AC02-78ET51013 and DE-ACO4-94AL85000.

\section{References}

\author{
Sandia is a multiprogram laboratory \\ operated by Sandia Corporation, a \\ Lockheed Martin Company, for the \\ United States Department of Energy \\ under contract DF:-ACO4-94AL85000.
}

[1] E.S. Marmar. Effects of wall conditioning and impurity levels on alcator c-mod discharge evolution. Proceedings. American Physical Society, 1993.

[2] B. LaBombard et al. Novel trace impurity injection. these proceedings, 1998.

[3] Y. Sakamoto, Y. Ishibe, K. Yano, et al. Electron cyclotron resonance discharge cleaning of jft-2 tokamak. Journal of Nuclear Materials, 93,94:333--337, 1980.

[4] P.K. Sharma, J.P. Singh, and D. Bora. Experimental study of a microwave produced toroidal plasma. Plasma Physics and Controlled Fusion, 39:1669-1680, 1997.

[5] B.V. Mech, A.A. Haasz, and J.W. Davis. Chemical erosion of pyrolitic graphite by low-energy $\mathrm{H}^{+}$and $\mathrm{D}^{+}$impact. J. Nuclear Mat., 241-243:1147-1151, 1997. See Fig. 1.

[6] W. Eckstein and V. Philipps. Physical Processes of the Interaction of Fusion Plasmas with Solids, chapter Physical Sputtering and Radiation-Enhanced Sublimation, page 114. Academic Press, 1996. See Fig. 4 on p. 103 and Fig. 12 on p. 114. 


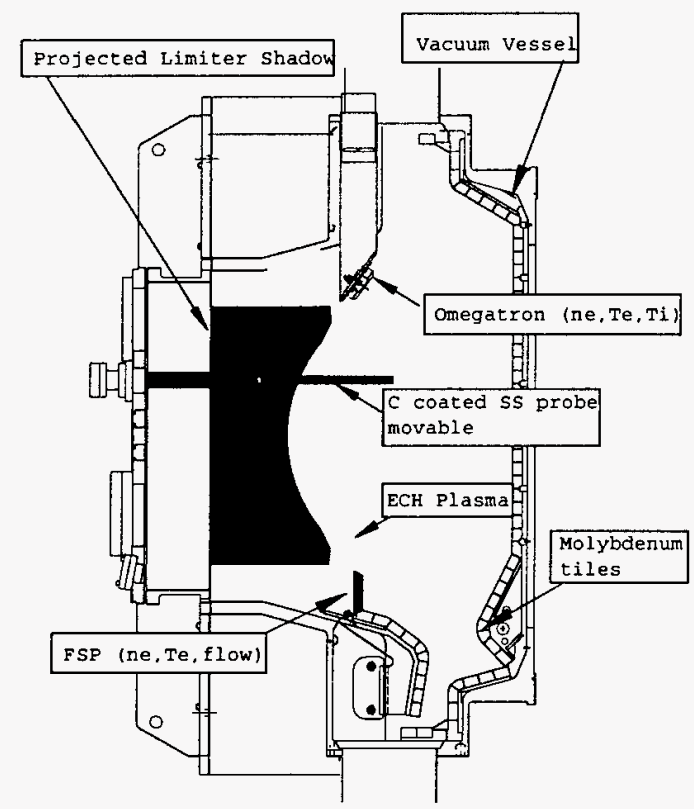

Figure 1: Poloidal projection of Alcator CMod tokamak. ECH plasmas (gray region) are swept in major radius. The Omegatron and FSP probe systems record local plasma conditions at the same toroidal location. A sample is coated with diamondlike carbon, is mounted on an assembly near the midplane, and is inserted radially into the plasma for erosion experiments. The sample assembly is located 180 degrees toroidally away from the Omegatron and FSP. 


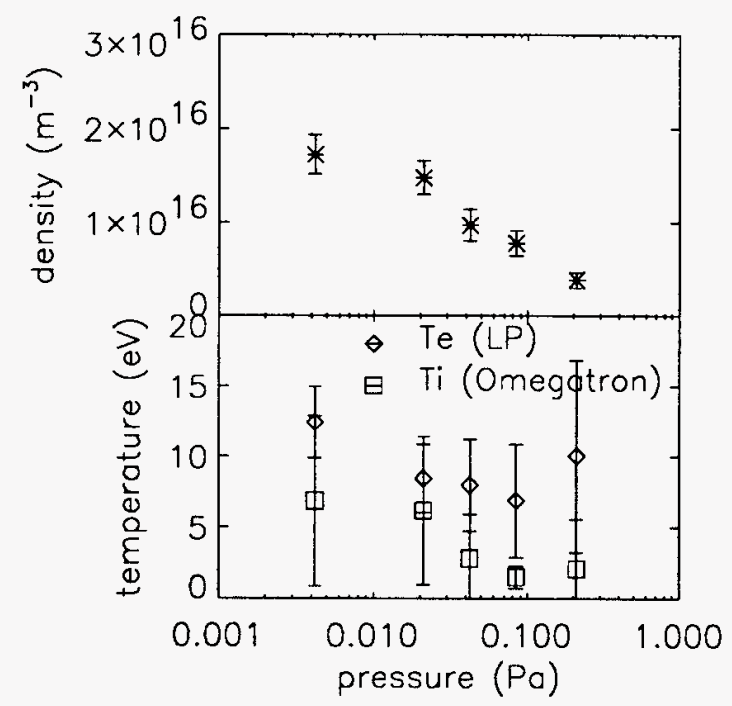

Figure 2: ECDC plasma density, electron and ion temperatures as functions of neutral deuterium pressure; resonance location is held fixed at $R_{\text {res }}=0.52 \mathrm{~m}$.

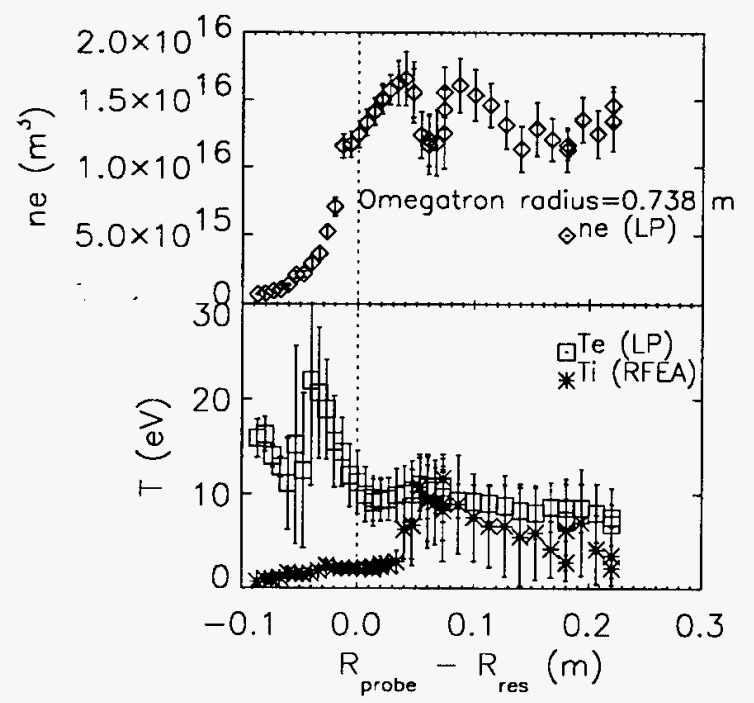

Figure 3: Effective radial profiles of ECDC plasma density, electron temperature, and ion temperature, measured by Omegatron probe. Major radius of measuring probe is held fixed, plasma electron cyclotron resonance moved. Ion temperature obtained from retarding field energy analysis; electron density and temperatures obtained from Langmuir probes. 


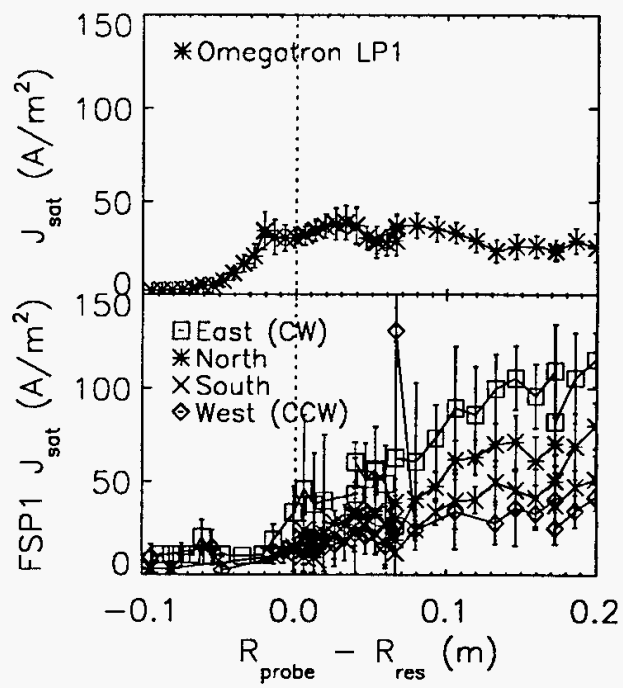

Figure 4: Effective radial profiles of ion saturation current density, measured by Langmuir probe LP1 on Omegatron probe and the four Langmuir probes on Fast Scanning Probe 1. Note the plasma flow indicated by the toroidal asymmetry in saturation current density. "East" points toroidally clockwise, viewed from top.

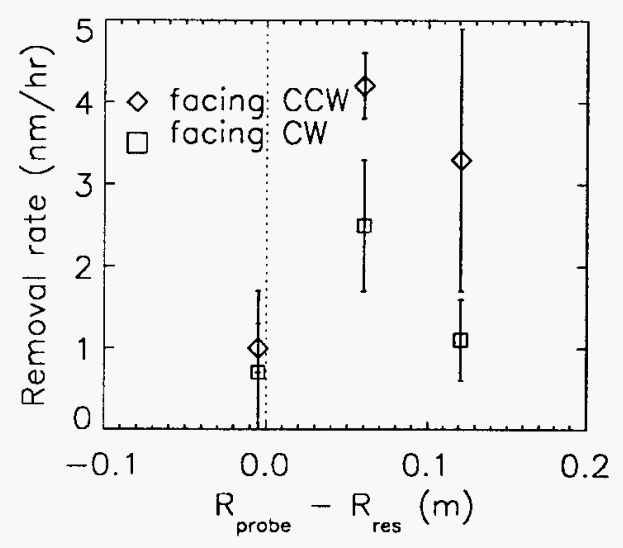

Figure 5: Removal rate of diamond-like carbon coating on stainless steel sample, as a function of the distance from cyclotron resonance location of the ECDC plasma. 

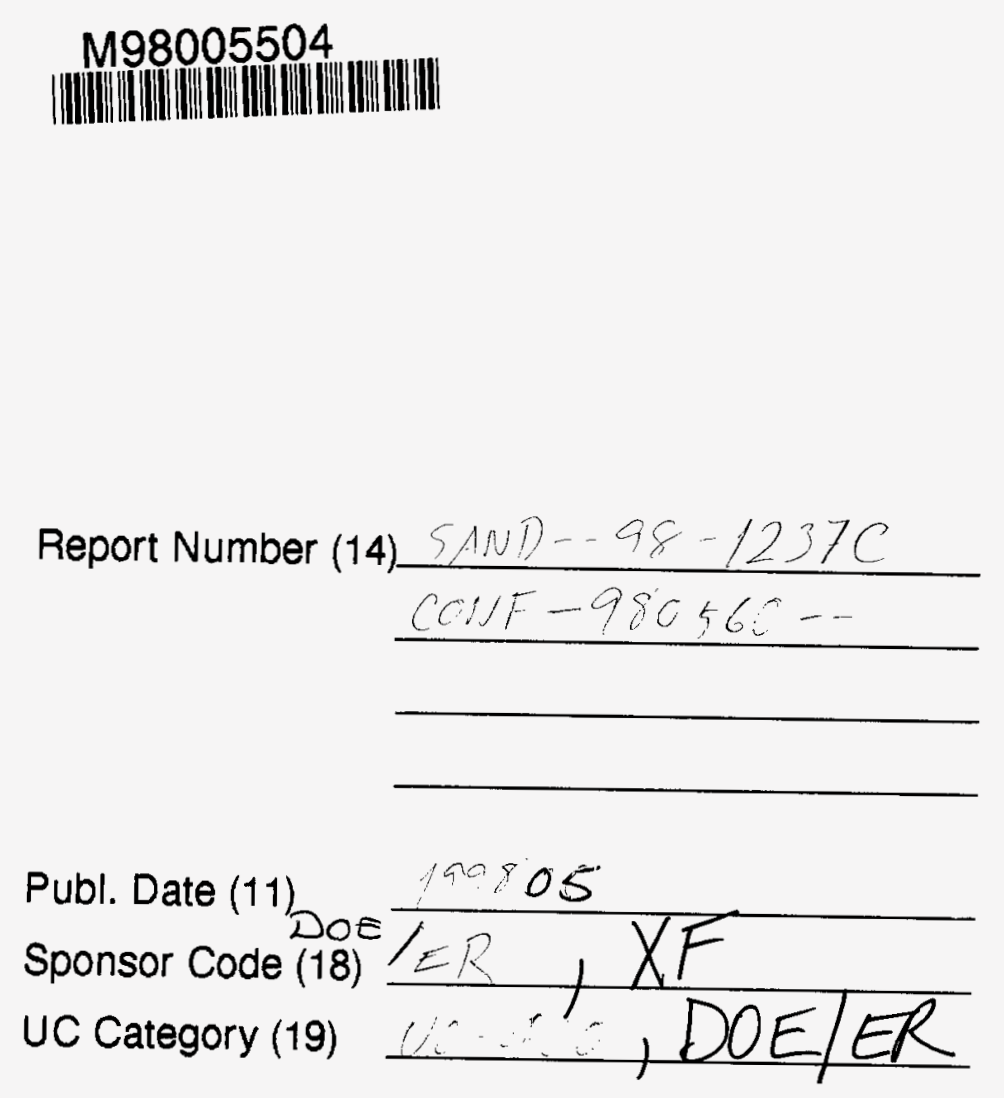

\section{2}

\section{DTIC QUALITY IVQPECTED I}

